\title{
Experimental test of the new analytic matrix formalism for spin dynamics
}

\author{
V. S. Morozov, A. W. Chao, A. D. Krisch, M. A. Leonova, R. S. Raymond, D. W. Sivers, and V. K. Wong \\ Spin Physics Center, University of Michigan, Ann Arbor, Michigan 48109-1120, USA \\ R. Gebel, A. Lehrach, B. Lorentz, R. Maier, D. Prasuhn, and H. Stockhorst \\ Forschungszentrum Jülich, Institut für Kernphysik, Postfach 1913, D-52425 Jülich, Germany \\ F. Hinterberger and K. Ulbrich \\ Helmholtz-Institut für Strahlen- und Kernphysik, Universität Bonn, D-53115 Bonn, Germany
}

A. Schnase

JAEA/J-PARC, Ring RF Group, Shirakata-Shirane 2-4, Tokai-Mura, Ibaraki 319-1195, Japan

(Received 8 February 2007; published 18 April 2007)

\begin{abstract}
We recently started testing Chao's proposed new matrix formalism for describing the spin dynamics due to a single spin resonance. The Chao formalism is probably the first fundamental improvement of the Froissart-Stora equation in that it allows analytic calculations of the beam polarization's behavior inside a resonance. We tested the Chao formalism using a $1.85 \mathrm{GeV} / c$ polarized deuteron beam stored in COSY, by sweeping an rf dipole's frequency through $200 \mathrm{~Hz}$, while varying the distance from the sweep's end frequency to an rf-induced spin resonance's central frequency. Since the Froissart-Stora equation itself can make no prediction inside a resonance, we compared our experimental data with the predictions of the Chao formalism and those of an empirical two-fluid model based on the Froissart-Stora equation. The data strongly favor the Chao formalism.
\end{abstract}

DOI: 10.1103/PhysRevSTAB.10.041001

PACS numbers: 29.27.Bd, 29.27.Hj, 41.75.Ak

\section{INTRODUCTION}

There has been considerable interest in polarized scattering experiments at storage rings such as the MIT-Bates Storage Ring [1], COSY [2], RHIC at Brookhaven [3], and HERA at DESY [4,5]. Many such polarized beam experiments benefit from the ability to precisely control the beam's polarization. The polarization of a stored beam can be manipulated in a well-controlled way by ramping an rf magnet's frequency through an rf-induced spin resonance. The Froissart-Stora formula [6] has been widely used to relate the beam polarizations before and after crossing a resonance. However, it is only valid for a constant-rate linear crossing from far below to far above the spin resonance. A matrix formalism was recently proposed by Chao [7] to treat many experimental conditions that the Froissart-Stora formula cannot treat. The Chao formalism can be used to calculate the polarization at any point inside an arbitrary piecewise linear crossing pattern. Thus, it allows one to calculate the spin dynamics when spin manipulating stored polarized beams outside the Froissart-Stora validity region. Our experiment tested the Chao formalism, using a $1.85 \mathrm{GeV} / c$ vertically polarized deuteron beam stored in COSY, by sweeping an rf dipole's frequency near or through an rf-induced spin resonance.

In an ideal flat circular storage ring or accelerator, with no horizontal bending magnetic fields, each particle's spin precesses around the vertical magnetic fields of the ring's bending dipoles. The spin tune $\nu_{s}$, which is the number of spin precessions during one turn around the ring, is proportional to the particle's energy

$$
\nu_{s}=G \gamma,
$$

where $G=(g-2) / 2$ is its gyromagnetic anomaly (for the deuteron $G_{d}=-0.142987$ ) and $\gamma$ is its Lorentz energy factor. The vertical polarization can be perturbed by an $\mathrm{rf}$ magnet's horizontal rf magnetic field. This perturbation can induce an rf depolarizing resonance $[6,8,9]$, which can be used to spin manipulate the stored polarized particles [10-24], such as deuterons. The rf-induced spin resonance's frequency is

$$
f_{r}=f_{c}\left(k \pm G_{d} \gamma\right)
$$

where $f_{c}$ is the deuteron's circulation frequency and $k$ is an integer.

Ramping an rf magnet's frequency through a spin resonance with strength $\epsilon$ can flip the stored beam's polarization. When the rf frequency is ramped at a constant rate by a range $\Delta f$, from far below to far above a resonance, during a ramp time $\Delta t$, the Froissart-Stora equation [6] can relate the beam's initial polarization $P_{i}$ to its final polarization $P$ after crossing the resonance,

$$
P=P_{i}\left\{2 \exp \left[-\frac{\left(\pi \epsilon f_{c}\right)^{2}}{\Delta f / \Delta t}\right]-1\right\} .
$$




\section{CHAO FORMALISM PREDICTION}

The paper [7] by Chao developed a matrix formalism for describing the spin dynamics during the crossing of an isolated spin resonance in a synchrotron. The formalism was developed by analytically solving the spinor equation of motion near an isolated spin resonance for two cases: (i) a constant distance between the spin tune $\nu_{s}=G \gamma$ and the rf-induced resonance tune $\nu_{\text {rf }} \equiv k \pm f_{\text {rf }} / f_{c}$; (ii) a linearly changing distance between $\nu_{s}$ and the instantaneous $\nu_{\mathrm{rf}}$. For each case a time-dependent matrix describing the spinor evolution was obtained. If a spin resonance is crossed with a piecewise linear crossing pattern, matrices corresponding to the individual linear segments can be multiplied sequentially to find the final spinor state, which determines the polarization.

To test the Chao formalism, we devised the experiment illustrated in Fig. 1. The frequency of an rf dipole was ramped over a frequency range $\Delta f$, which started at a frequency $f_{\text {start }}$ away from a spin resonance and ended at a frequency $f_{\text {end }}$ near or sometimes inside the resonance, which was centered at $f_{r}$. The frequency range $\Delta f$ and ramp time $\Delta t$ were both held fixed, while $f_{\text {start }}$ and therefore $f_{\text {end }}$ were varied. The rf dipole was turned off abruptly at $f_{\text {end }}$ to preserve the vertical polarization component at the instant of the turn off. The beam's vertical polarization was then measured.

For the experiment shown in Fig. 1, the final spinor state is given by [7]

$$
\left[\begin{array}{l}
h \\
g
\end{array}\right]_{\theta_{\text {end }}}=U_{\Gamma, \theta_{c}, \epsilon}\left(\theta_{\text {end }}, \theta_{\text {start }}\right)\left[\begin{array}{l}
h \\
g
\end{array}\right]_{\theta_{\text {start }}},
$$

where $h$ and $g$ are the spinor components, $\theta_{\text {start }}$ and $\theta_{\text {end }}$ are the particle's "times" $\left(\theta \equiv 2 \pi f_{c} t\right)$ at the ramp's start and end, respectively, $\Gamma$ is the crossing rate, $\theta_{c}$ is the particle's

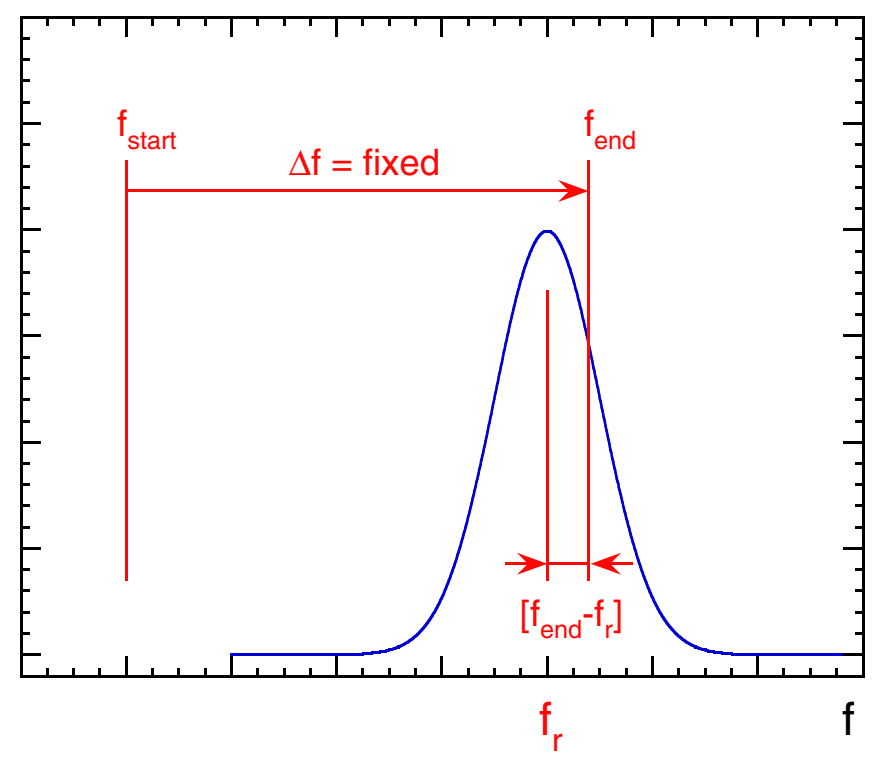

FIG. 1. (Color) Schematic of the Chao formalism test. time at the resonance crossing, $\epsilon$ is the resonance strength, and $U_{\Gamma, \theta_{c}, \epsilon}\left(\theta_{\text {end }}, \theta_{\text {start }}\right)$ is a $2 \times 2$ matrix given explicitly in Eq. (52) of Ref. [7]. The final vertical polarization $P$ is obtained from Eq. (11) of Ref. [7]:

$$
P=\left|h\left(\theta_{\text {end }}\right)\right|^{2}-\left|g\left(\theta_{\text {end }}\right)\right|^{2} .
$$

Since the ramp starts far from the spin resonance, the initial spinor is simply that of a pure vertical polarization.

We related the Chao parameters $\theta_{\text {start }}, \theta_{\text {end }}, \Gamma$, and $\theta_{c}$ to our experimental parameters $f_{c}, f_{r}, f_{\text {end }}, \Delta f$, and $\Delta t$ using

$$
\begin{gathered}
\theta_{\text {start }}=-\pi f_{c} \Delta t ; \\
\theta_{\text {end }}=\pi f_{c} \Delta t ; \\
\Gamma=\frac{1}{2 \pi f_{c}^{2}} \frac{\Delta f}{\Delta t} ; \\
\theta_{c}=\frac{1}{\Gamma} \frac{[\Delta f / 2]-\left[f_{\text {end }}-f_{r}\right]}{f_{c}} .
\end{gathered}
$$

We then used Eqs. (4) and (5) with the substitution of Eqs. (6)-(9) to obtain a prediction for the final vertical polarization $P$ as a function of $\left[f_{\text {end }}-f_{r}\right]$. The prediction for a particular set of experimental parameters is shown in Fig. 2 by the red solid line. Note the interesting predicted oscillations for positive values of $\left[f_{\text {end }}-f_{r}\right]$.

The red solid line in Fig. 2 is a single-particle prediction $\left(f_{r}\right.$ spread $\left.=0\right)$ that ignores the $f_{r}$ spread caused by the beam's momentum spread, which may be significant in a real beam. Thus, we included the $f_{r}$ spread by folding the single-particle prediction curve together with Gaussians representing different $f_{r}$ spreads. These predictions are shown in Fig. 2 by the different color dotted lines. Note that the $f_{r}$ spread smoothes the polarization oscillations; their amplitude is reduced as the $f_{r}$ spread increases.

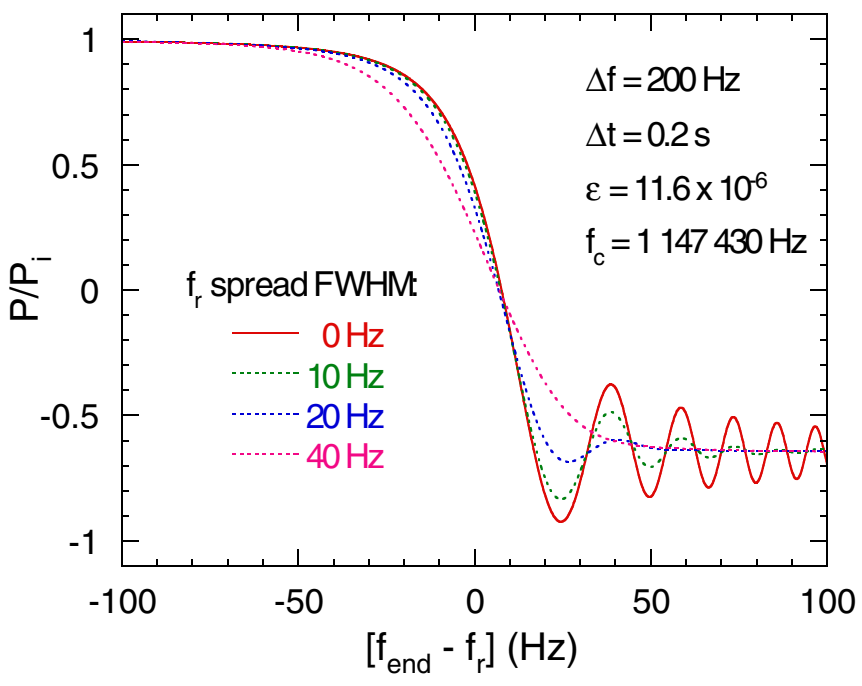

FIG. 2. (Color) Predictions of the Chao formalism for the study shown in Fig. 1. 


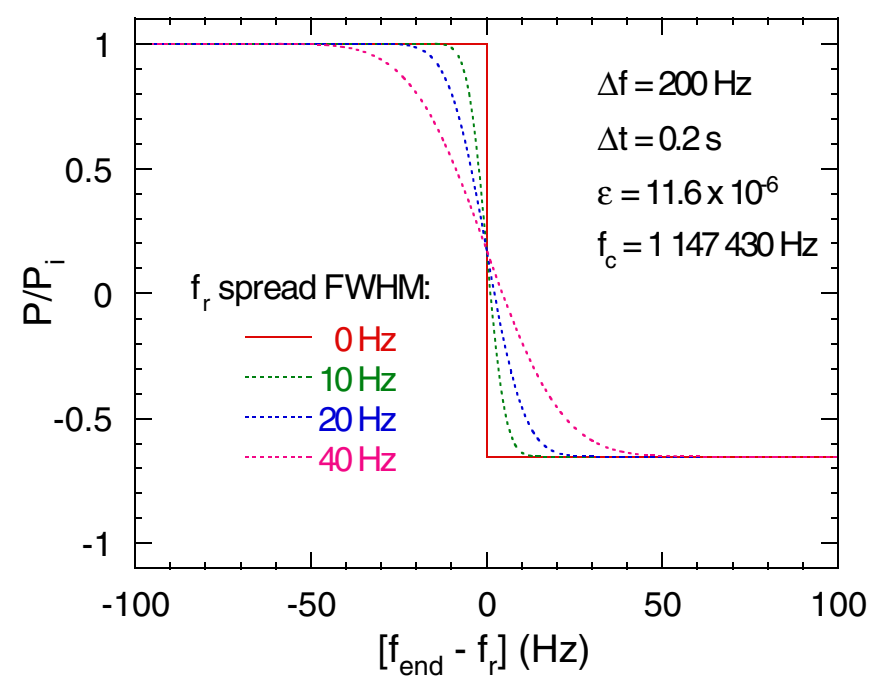

FIG. 3. (Color) Predictions of the two-fluid model for the study shown in Fig. 1.

\section{TWO-FLUID MODEL PREDICTION}

The Froissart-Stora formula equation (3) is not valid for the experiment shown in Fig. 1 because the spin resonance is not crossed completely. However, a two-fluid model based on the Froissart-Stora formula equation (3) may be useful in cases when the beam's $f_{r}$ spread is significantly greater than the single-particle resonance width $w=2 \epsilon f_{c}$ [10].

The model assumes that the frequency ramp only affects those beam particles with resonance frequencies within the ramp's frequency range $\left(f_{\text {start }}<f_{r}<f_{\text {end }}\right)$. The final polarization of these particles is obtained using Eq. (3), the Froissart-Stora formula. The model assumes that the remaining fraction of particles retains their initial polarization $P_{i}$. The two-fluid/FS polarization is the average of these two beam fractions' polarizations. Thus, for a beam with a density function $\rho\left(f_{r}\right)$, the beam's final polarization is

$$
\begin{aligned}
P= & P_{i}\left\{2 e^{-\left[\left(\pi \epsilon f_{c}\right)^{2} /(\Delta f / \Delta t)\right]}-1\right\} \overbrace{\int_{f_{\text {start }}}^{f_{\text {end }}} \rho\left(f_{r}\right) d f_{r}}^{\text {affected fraction }} \\
& +P_{i} \underbrace{\left\{1-\int_{f_{\text {start }}}^{f_{\text {end }}} \rho\left(f_{r}\right) d f_{r}\right\}}_{\text {unaffected fraction }} \cdot
\end{aligned}
$$

Assuming a Gaussian distribution of the $f_{r}$ spread, we used Eq. (10) to obtain predictions for a few different widths of the $f_{r}$ spread. These predictions are plotted in Fig. 3.

\section{APPARATUS}

The apparatus used for this experiment, including the COSY storage ring [25-28], the EDDA detector [29,30], the rf dipole, the electron cooler [31], the low energy polarimeter [32], the injector cyclotron, and the polarized ion source [33-35] are shown in Fig. 4. The beam emerging from the polarized $D^{-}$ion source was accelerated by the cyclotron to COSY's deuteron injection energy of about $75.7 \mathrm{MeV}$. Then the low energy polarimeter measured the beam's polarization before injection into COSY to monitor the stable operation of the cyclotron and ion source. The $D^{-}$beam was next strip injected into COSY.

For the Chao formalism test, we used the electron cooler at injection energy to reduce the beam's size and momentum spread. A $20.6 \mathrm{keV}$ electron beam cooled the deuteron beam to its equilibrium emittances in both the longitudinal and transverse dimensions. The beam was then accelerated to the experimental momentum of $1.85 \mathrm{GeV} / c$. The $\mathrm{rf}$ acceleration cavity was turned off and shorted during COSY's flattop; thus, there were no synchrotron oscillations.

We manipulated the deuteron's polarization using a ferrite-core rf dipole, with an 8-turn copper coil, which produced a uniform radial magnetic field. The rf dipole was part of an RLC resonant circuit, which operated near $917 \mathrm{kHz}$, typically at an rf voltage of $3.1 \mathrm{kV} \mathrm{rms}$ producing an $\mathrm{rf} \int B_{\mathrm{rms}} d l$ of $0.60 \pm 0.03 \mathrm{~T} \mathrm{~mm}$.

The EDDA detector $[29,30]$ was used to measure the beam's polarization in COSY. We reduced its systematic

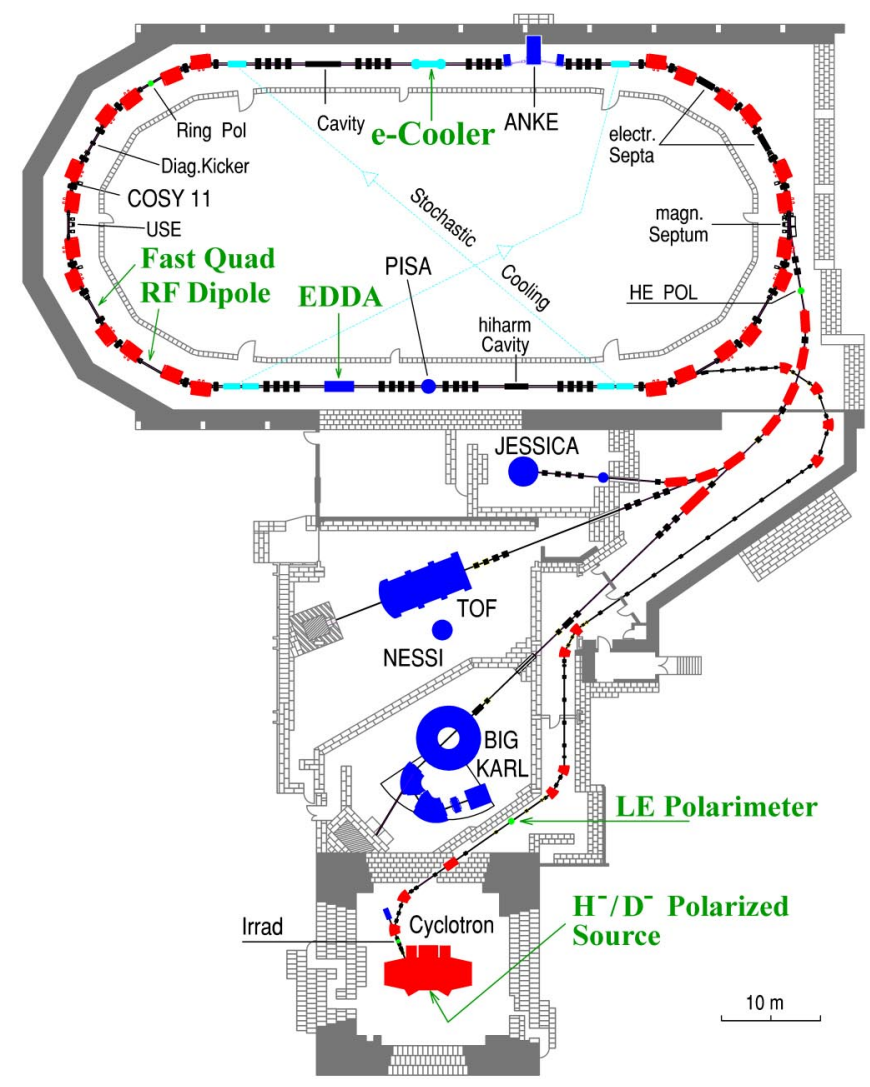

FIG. 4. (Color) Layout of the COSY storage ring, with its injector cyclotron and polarized ion source. Also shown are the EDDA detector, the rf dipole, the electron cooler, and the low energy polarimeter. 
errors by cycling the beam through 4 different vector and tensor vertical polarization states:

$$
\left(P_{V}, P_{T}\right)=(0,0),(+1,+1),\left(\frac{1}{3},-1\right),\left(-\frac{2}{3}, 0\right) .
$$

The measured $(+1,+1)$ vector polarization, before spin manipulation, was about $63 \%$.

\section{EXPERIMENTAL TESTS}

The deuteron circulation frequency in the COSY ring was $f_{c}=1.14743 \mathrm{MHz}$ at $1.850 \mathrm{GeV} / c$, where its Lorentz energy factor was $\gamma=1.4046$. With these parameters, Eq. (1) gave a spin tune $\nu_{s}=G \gamma$ of -0.20084 . Thus, at $1.850 \mathrm{GeV} / c$, Eq. (2) implies that the $k=1$ spin resonance's central frequency should occur at

$$
f_{r}=(1+G \gamma) f_{c}=917.0 \mathrm{kHz} .
$$

We experimentally determined $f_{r}$ and the resonance's full width at half maximum (FWHM) $w$ by measuring the polarization, after running the rf dipole at different fixed frequencies near $917.0 \mathrm{kHz}$. In this study [36] $\epsilon$ was set at about $1.4 \times 10^{-6}$; we obtained $f_{r}=916992 \pm 10 \mathrm{~Hz}$ and $w=23 \pm 2 \mathrm{~Hz}$. This measured width $w$ was dominated by the $f_{r}$ spread due to the beam's $\Delta p / p$ since the resonance's natural width $\left(2 \epsilon f_{c}\right)$ was only $\sim 3 \mathrm{~Hz}$.

We calibrated the strength of an rf-dipole-induced spin resonance against the rf dipole's voltage. This involved ramping the rf dipole's frequency through the resonance with various ramp times $\Delta t$ while keeping the rf dipole's frequency range $\Delta f$ and its voltage fixed; then we measured the final polarization after each frequency ramp. We found the strength $\epsilon$ by fitting these data to Eq. (3) with $\epsilon$ as a fit parameter [36].

To experimentally test the Chao formalism, we ramped the rf dipole's frequency over a range $\Delta f$, which started at $f_{\text {start }}$ and ended at $f_{\text {end }}$ near the $\mathrm{rf}$ resonance frequency $f_{r}$, as shown in Fig. 1. After reaching $f_{\text {end }}$, the rf dipole was turned off abruptly in a few $\mu$ s. We then measured the beam's final polarization. This procedure was repeated at different values of $f_{\text {end }}$ while holding fixed: the frequency ramp range at $\Delta f=200 \mathrm{~Hz}$, the ramp time at $\Delta t=4 \mathrm{~s}$, and the resonance strength at $\epsilon=9.56 \times 10^{-6}$. The measured polarization ratio, averaged for all spin states, is plotted against $\left[f_{\text {end }}-f_{r}\right]$ in Fig. 5. Predictions of the Chao formalism for 0 and $23 \mathrm{~Hz}$ FWHM Gaussian $f_{r}$ spreads and of the two-fluid model are shown in Fig. 5 by the dotted red, solid green, and dashed blue lines, respectively. To compare how well the different predictions agree with the data, we calculated $\chi^{2} / N$ for each prediction. The $\chi^{2}$ analysis included only the data's statistical errors and ignored systematic errors; thus, the $\chi^{2} / N$ values were rather large. Note that the fits of all three predictions are extremely sensitive to the value of $f_{r}$, as shown in Fig. 8(a). Thus, we chose $f_{r}=916994 \mathrm{~Hz}$, which simul-

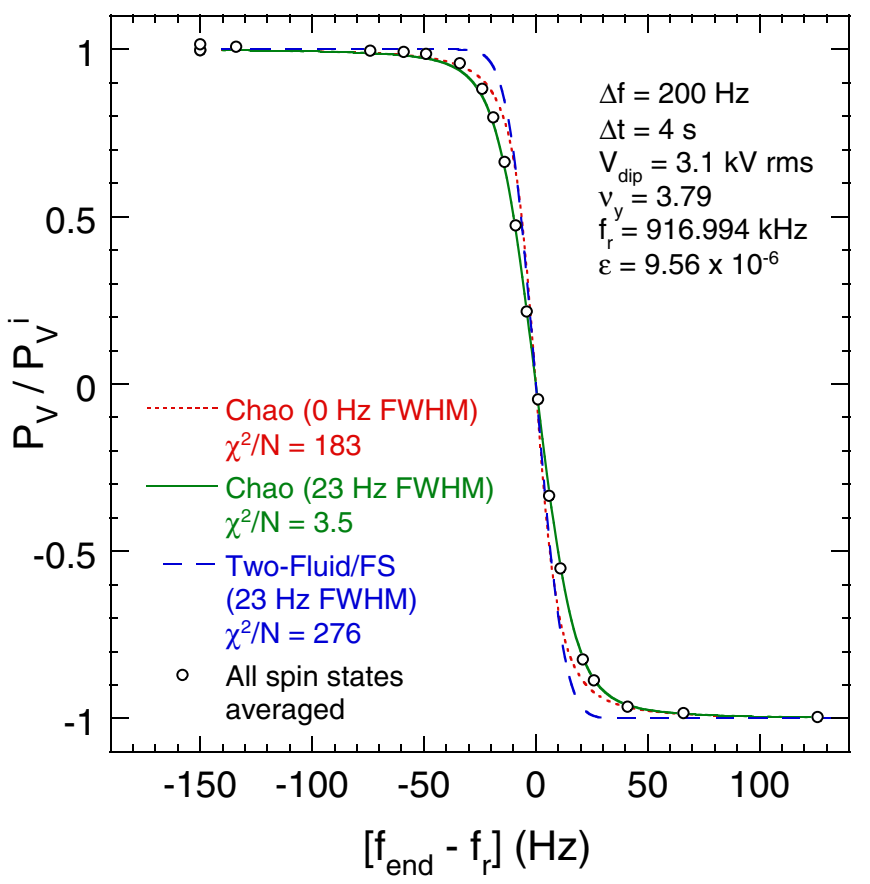

FIG. 5. (Color) The measured vector polarization ratio at $1850 \mathrm{MeV} / c$, averaged for all spin states, is plotted vs the difference between the rf-dipole frequency sweep's ending frequency $f_{\text {end }}$ and the spin resonance's central frequency $f_{r}$. The rf dipole's frequency ramp time $\Delta t$ was $4 \mathrm{~s}$; its frequency range $\Delta f$ was $200 \mathrm{~Hz}$, and its $\epsilon$ was $9.56 \times 10^{-6}$. The electron cooling was on. The dotted red, solid green, and dashed blue lines are predictions of the Chao formalism for 0 and $23 \mathrm{~Hz}$ FWHM Gaussian $f_{r}$ spreads and of the two-fluid model, respectively. The data errors, used in calculating $\chi^{2} / N$, are purely statistical and are smaller than the symbol size.

taneously minimizes the $\chi^{2} / N$ for all three predictions and is certainly consistent with the $916992 \pm 10 \mathrm{~Hz}$ measured earlier [36]. The $\chi^{2}$ analysis in Fig. 5 strongly favors the Chao formalism prediction for the measured $23 \mathrm{~Hz}$ FWHM $f_{r}$ spread.

We next did a similar study with the rf dipole's ramp time $\Delta t$ set at $0.2 \mathrm{~s}$. This faster ramp resulted in an onlypartial spin flip when the resonance was fully crossed. These data are shown in Fig. 6, which also shows the Chao formalism and two-fluid model predictions.

A blowup of the region in Fig. 6, where the oscillations were expected, is shown in Fig. 7. Most data points fall almost exactly on top of the green solid line, supporting the validity of the $23 \mathrm{~Hz}$ prediction of the Chao formalism. With no $f_{r}$ spread, the Chao formalism predicts largeamplitude oscillations of the polarization. However, the $f_{r}$ spread smoothes these oscillations leaving only a small wiggle in the predicted polarization. Note that we chose $f_{r}=916.987 \mathrm{kHz}$ because, as shown in Fig. 8, it was the only $f_{r}$ value, which gave a $\chi^{2} / N$ minimum below 100 for any of the three predictions for either Fig. 6 [see Fig. 8(b)] or Fig. 7 [see Fig. 8(c)]. (Note that Fig. 8 suggests that there 


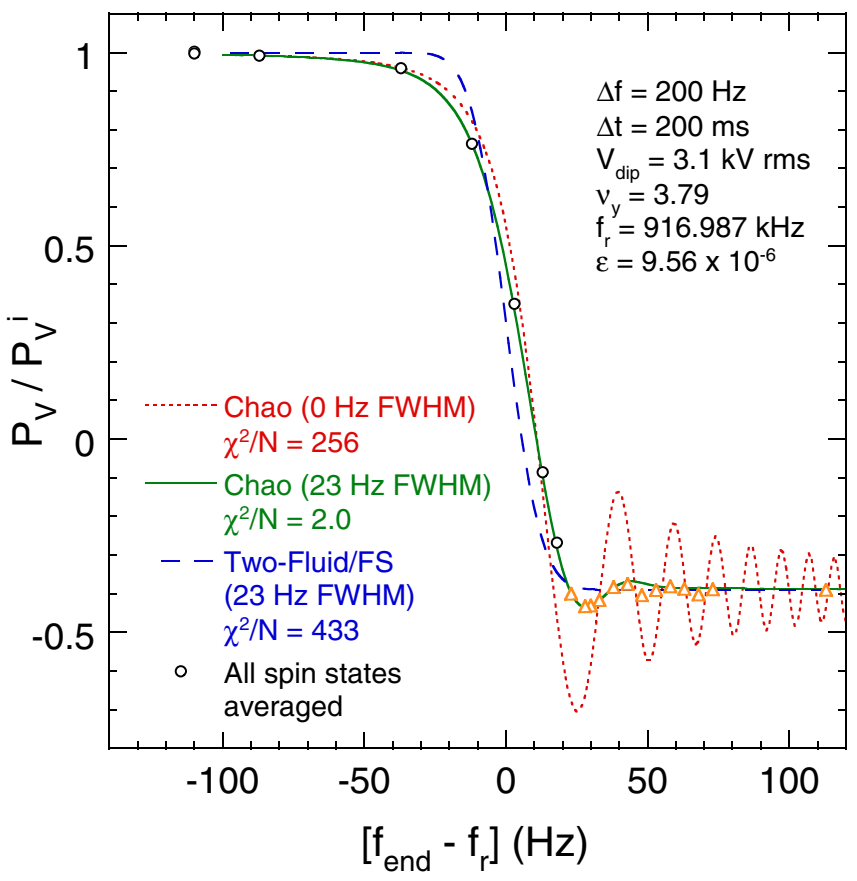

FIG. 6. (Color) The measured vector polarization ratio is again plotted vs $\left[f_{\text {end }}-f_{r}\right]$ as in Fig. 5. However, now the rf dipole's frequency ramp time $\Delta t$ was $0.2 \mathrm{~s}$. The $\chi^{2} / N$ is calculated only for the black circles at $f_{\text {end }}-f_{r}<+20 \mathrm{~Hz}$. The data at $f_{\text {end }}-f_{r}>+20 \mathrm{~Hz}$ (shown as triangles) are analyzed in Fig. 7.

was a $7 \mathrm{~Hz}$ shift in $f_{r}$ between taking the data in Fig. 5 and the data in Figs. 6 and 7. A $7 \mathrm{~Hz}$ shift is consistent with COSY's stability level on its flattop with the rf cavity off.) The $\chi^{2}$ analysis of the Fig. 6 and the Fig. 7 data, with this

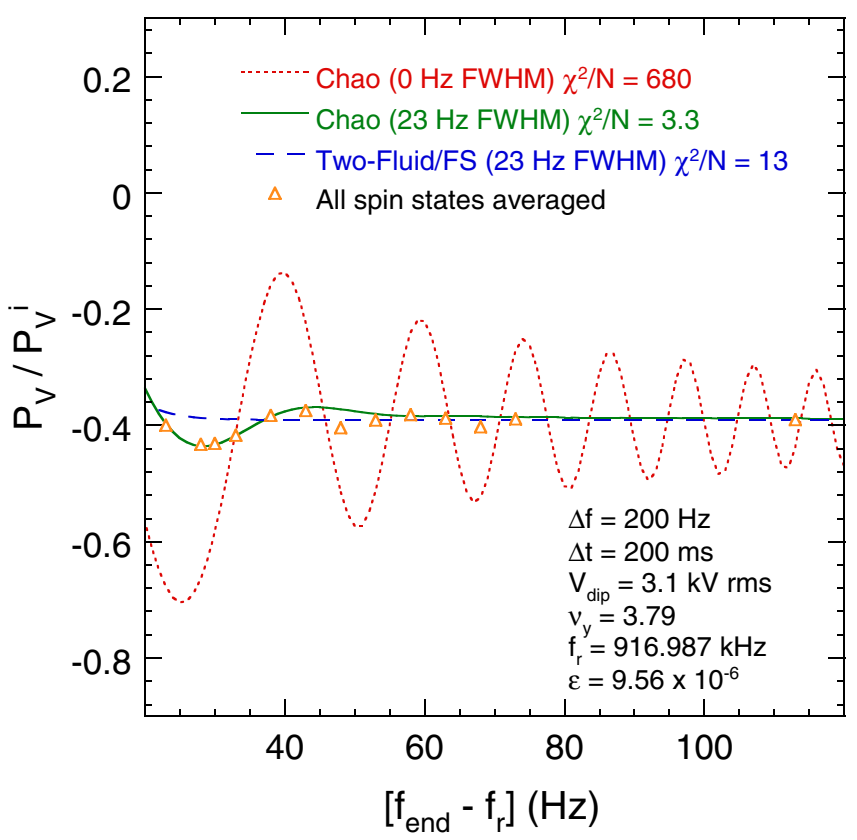

FIG. 7. (Color) The measured vector polarization ratio is again plotted vs $\left[f_{\text {end }}-f_{r}\right]$ as in Fig. 6. However, now the scale is expanded to show only the region of predicted oscillations.

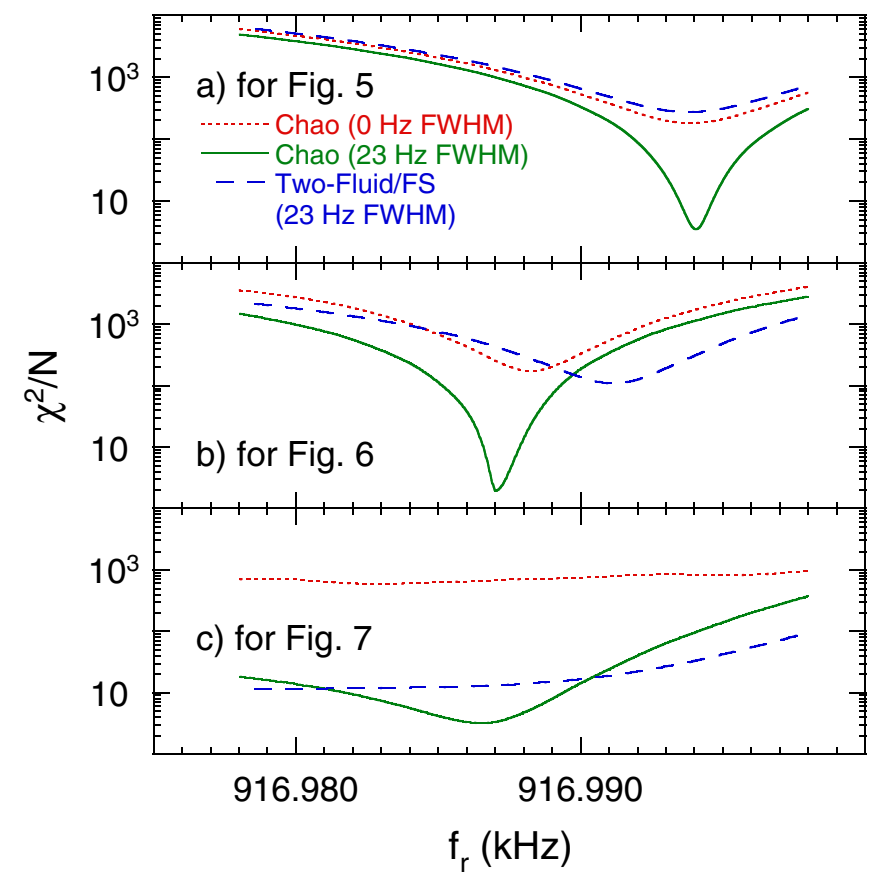

FIG. 8. (Color) The $\chi^{2} / N$ values for the three predictions are plotted vs $f_{r}$ for Figs. 5-7 as indicated above.

$f_{r}$ value, favors the $23 \mathrm{~Hz}$ Chao formalism prediction even more strongly than the Fig. 5 data.

To further improve the Chao test, we made a prediction for the study shown in Fig. 1 using a 4 times faster crossing rate than in Figs. 6 and 7. This $\Delta t=100$ ms prediction is shown in Fig. 9; note that folding in the $23 \mathrm{~Hz}$ FWHM Gaussian now only partly smoothes the oscillations in Fig. 9 because their period is now longer due to the faster crossing rate. For the $23 \mathrm{~Hz}$ FWHM $f_{r}$ spread, the predicted maximum peak-to-peak amplitude of the oscilla-

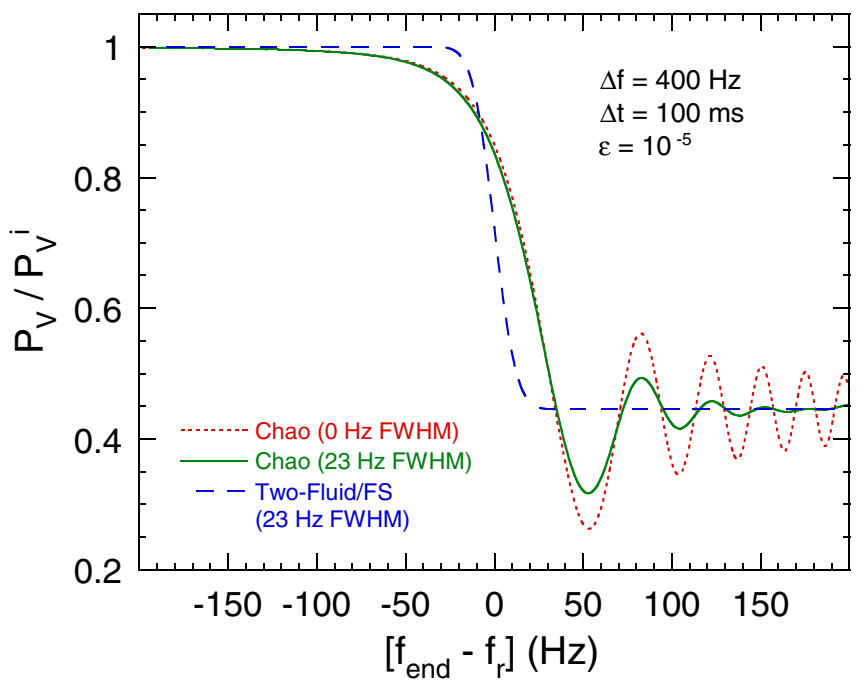

FIG. 9. (Color) The predicted vector polarization ratio is plotted vs $\left[f_{\text {end }}-f_{r}\right]$ as in Fig. 5 . However, now $\Delta f$ is $400 \mathrm{~Hz}$ and $\Delta t$ is $100 \mathrm{~ms}$ while the resonance strength $\epsilon$ is almost identical. 
tions is about $15 \%$; this should allow a more convincing test of the Chao formalism. We plan to soon test this prediction at COSY.

\section{SUMMARY}

We used $1.85 \mathrm{GeV} / c$ vertically polarized deuterons stored in COSY to experimentally test the recently proposed Chao matrix formalism for describing the spin dynamics during crossing of an isolated spin resonance in a synchrotron. The Chao formalism allows predictions for experiments where the Froissart-Stora formula is not valid. We conducted such an experiment at COSY by ramping an rf dipole's frequency through a range ending near a spin resonance; both the frequency range and ramp time were fixed while we varied the ramp's start and therefore end frequencies. We compared our experimental data with the predictions of the Chao formalism and of the two-fluid model. Our data strongly favor the validity of the Chao formalism for the measured [36] resonance width of $23 \mathrm{~Hz}$.

\section{ACKNOWLEDGMENTS}

We would like to thank the entire COSY staff for the successful operation of COSY with its injector cyclotron and polarized ion source. We are grateful to E. D. Courant, Ya. S. Derbenev, D. Eversheim, G. Fidecaro, W. Haeberli, H. Huang, H. Rohdjeß, T. Roser, H. Sato, W. Scobel, $\mathrm{K}$. Yonehara, and others for their help and advice. This research was supported by grants from the German BMBF Science Ministry.

[1] R. Alarcon et al. (BLAST Collaboration), in MIT Bates Report No. 2-39, 1999.

[2] H. Rohdjess et al., in SPIN 2002: 15th International Spin Physics Symposium, Upton, NY, 2002, edited by Y.I. Makdisi et al., AIP Conf. Proc. No. 675 (AIP, Melville, New York, 2003), p. 523.

[3] Y. Makdisi, in High Energy Spin Physics: 11th International Symposium, Bloomington, IN, 1994, edited by Kenneth J. Heller and Sandra L. Smith, AIP Conf. Proc. No. 343 (AIP, Woodbury, NY, 1995), p. 75.

[4] A. D. Krisch et al. (SPIN Collaboration), University of Michigan Report No. UM-HE 96-20, 1996; University of Michigan Report No. UM-HE 99-05, 1999.

[5] A. Airapetian et al. (HERMES Collaboration), DESYPRC Report No. 99-08, 1999.

[6] M. Froissart and R. Stora, Nucl. Instrum. Methods 7, 297 (1960).

[7] A. W. Chao, Phys. Rev. ST Accel. Beams 8, 104001 (2005).

[8] E. D. Courant, Bull. Am. Phys. Soc. 7, 33 (1962); Report No. BNL-EDC-45, 1962.

[9] B. W. Montague, Phys. Rep. 113, 35 (1984).

[10] D. D. Caussyn et al., Phys. Rev. Lett. 73, 2857 (1994).

[11] D. A. Crandell et al., Phys. Rev. Lett. 77, 1763 (1996).

[12] B. von Przewoski et al., Rev. Sci. Instrum. 67, 165 (1996).
[13] B. B. Blinov et al., Phys. Rev. Lett. 81, 2906 (1998); V. A. Anferov et al., in Proceedings of the 13th International Symposium on High Energy Spin Physics (IHEP), Protvino, Russia, 1998, edited by N.E. Tyurin et al. (World Scientific, Singapore, 1999), p. 503.

[14] V.A. Anferov et al., Phys. Rev. ST Accel. Beams 3, 041001 (2000).

[15] B. B. Blinov et al., Phys. Rev. ST Accel. Beams 3, 104001 (2000).

[16] A. M. T. Lin et al., in SPIN 2000: 14th International Spin Physics Symposium, Osaka, 2000, edited by K. Hatanaka, AIP Conf. Proc. No. 570 (AIP, Melville, New York, 2001), p. 736.

[17] V.S. Morozov et al., Phys. Rev. ST Accel. Beams 4, 104002 (2001).

[18] B. B. Blinov et al., Phys. Rev. Lett. 88, 014801 (2002); V.S. Morozov et al., in SPIN 2002: 15th International Spin Physics Symposium, Upton, NY, 2002, in Ref. [2], p. 776.

[19] V. S. Morozov et al., Phys. Rev. Lett. 91, 214801 (2003).

[20] K. Yonehara et al., in Proceedings of the 8th Conference on the Intersections of Particle and Nuclear Physics, New York, NY, 2003, edited by Z. Parsa, AIP Conf. Proc. No. 698 (AIP, Melville, NY, 2003), p. 763.

[21] M. A. Leonova et al., Phys. Rev. Lett. 93, 224801 (2004).

[22] V.S. Morozov et al., Phys. Rev. ST Accel. Beams 7, 024002 (2004).

[23] V.S. Morozov et al., Phys. Rev. ST Accel. Beams 8, 061001 (2005).

[24] M. A. Leonova et al., Phys. Rev. ST Accel. Beams 9, 051001 (2006).

[25] R. Maier, Nucl. Instrum. Methods Phys. Res., Sect. A 390, 1 (1997).

[26] A. Lehrach et al., in Proceedings of the 1999 Particle Accelerator Conference, New York, NY, 1999, edited by A. Luccio and W. MacKay (IEEE, Piscataway, NJ, 1999), p. 2292.

[27] H. Stockhorst et al., in Proceedings of the 8th European Particle Accelerator Conference, Paris, 2002 (EPS-IGA/ CERN, Geneva, 2002), p. 629.

[28] A. Lehrach et al., in SPIN 2002: 15th International Spin Physics Symposium, Upton, NY, 2002, in Ref. [2], p. 153.

[29] V. Schwarz et al., in Proceedings of the 13th International High Energy Spin Physics Symposium, Protvino, 1998, in Ref. [13], p. 560.

[30] M. Altmeier et al. (EDDA Collaboration), Phys. Rev. Lett. 85, 1819 (2000).

[31] H. Stein et al., At. Energ. 94, 24 (2003).

[32] D. Chiladze et al., Phys. Rev. ST Accel. Beams 9, 050101 (2006).

[33] P.D. Eversheim et al., in Proceedings of the 8th International Symposium on Polarization Phenomena in Nuclear Physics, Bloomington, IN, 1994, edited by Edward J. Stephenson and Steven E. Vigdor, AIP Conf. Proc. No. 339 (AIP, Woodbury, NY, 1995), p. 668.

[34] R. Weidmann et al., Rev. Sci. Instrum. 67, 1357 (1996).

[35] O. Felden et al., in Proceedings of the 9th International Workshop on Polarized Sources and Targets, Nashville, IN, 2001, edited by V. P. Derenchuk and B. von Przewoski (World Scientific, Singapore, 2002), p. 200.

[36] A. D. Krisch et al. (unpublished). 